\title{
On spectra of hyperbolic surfaces without thin handles
}

\author{
M.B. Dubashinskiy*
}

January 8, 2019

\begin{abstract}
We obtain a sharp lower estimate on eigenvalues of Laplace-Beltrami operator on a hyperbolic surface with injectivity radius bounded from the below.
\end{abstract}

\section{Introduction}

Let $\Omega$ be a hyperbolic surface, that is, a Riemannian manifold of real dimension 2 with constant Gaussian curvature -1 ; we assume that $\Omega$ is compact and has no border. Denote by $g$ the genus of $\Omega$. Let $\Delta$ be Laplace-Beltrami operator on $\Omega$; it has purely discrete spectrum since $\Omega$ is compact. Denote by $\lambda_{j}=\lambda_{j}(\Omega)$ the $j$ th eigenvalue of $-\Delta(j=0,1,2, \ldots)$. Our main result is the following

Theorem 1. Let $r>0$. There exists a constant $c(r)>0$ such that if injectivity radius of $\Omega$ is greater than $r$ then $\lambda_{\lceil\varepsilon g\rceil} \geq c(r) \cdot \varepsilon^{2}$ for any $\varepsilon \leq 2$.

In what follows, we denote by $c(r)$ any positive constant depending only on $r$ (but not on $\varepsilon$, $g$ and $\Omega$ ).

Proposition 8 below shows that our estimate is sharp in the order.

A theorem by Otal and Rosas ([3]) says that $\lambda_{2 g-2}>1 / 4$ for any $\Omega$ of genus $g$. To the other hand, for a given $\delta>0, N \in \mathbb{N}$ and $g=2,3, \ldots$ there exists a hyperbolic surface $\Omega$ of genus $g$ with $\lambda_{2 g-3}<\delta$ and $\lambda_{2 g-2+N}<1 / 4+\delta$. Validity of these inequalities is related to the existence of thin handles on $\Omega$ (see [1]). In other words, eigenvalues are small when injectivity radius of $\Omega$ degenerates. Theorem 1 gives the lower estimate on eigenvalues under the assumption on this radius.

Acknowledgments. The problem was stated by M. Mirzakhani. Author is also grateful to P.G. Zograf for introducing the topic.

\footnotetext{
* Chebyshev Laboratory, St. Petersburg State University, 14th Line 29b, Vasilyevsky Island, Saint Petersburg 199178, Russia.

e-mail: mikhail.dubashinskiy@gmail.com

Research is supported by the Russian Science Foundation grant 14-21-00035.

Keywords: hyperbolic surface, Laplace-Beltrami eigenvalues, Cheeger-Yau isoperimetric inequality.

MSC 2010: primary 35P15, secondary 58J50.
} 


\section{Proof of Theorem 1}

Our proof of Theorem 1 is a slight refinement of Buser's argument leading to the estimate $\lambda_{2 g-2} \geq 10^{-12}$ (see [1]) together with simple Lemma 7 on graphs.

We are going to apply Dirichlet-Neumann bracketing technique. Recall that if $X \subset \Omega$ is a set with positive area and piecewise smooth boundary, then its Cheeger constant is defined as

$$
h(X):=\inf \frac{l(A)}{\min \left\{|B|,\left|B^{\prime}\right|\right\}},
$$

where $A$ ranges over the family of all finite unions of piecewise smooth curves on $X$ cutting $X$ into two disjoint subsets $B$ and $B^{\prime}$. Here, $l(A)$ is length of $A$ and $|\cdot|$ is Riemannian volume on $\Omega$. A very standard combination of geometric implementation of minimax principle together with Cheeger-Yau isoperimetric inequality ([1], see also [4], [5]) leads to the following conclusion:

Theorem 2. Suppose that $k \in \mathbb{N}$ and that $\Omega$ is subdivided into union of sets $X_{1}, \ldots, X_{k}$ with piecewise-smooth boundaries and disjoint interiors. Then

$$
\lambda_{k}(\Omega) \geq \min _{j=1, \ldots, k} \frac{h^{2}\left(X_{j}\right)}{4} .
$$

An appropriate subdivision of $\Omega$ will be obtained via trianguation of controlled size. For this, recall a result by Buser (Theorem 4.5.2 in [1], see also [2]).

Definition 3. A closed domain $D \subset \Omega$ is called a trigon if $D$ is of one of the following two types:

1. D a simply connected embedded geodesic triangle (an ordinary triangle);

2. $D$ is embedded doubly connected domain bounded by a geodesic cycle and two geodesic arcs (a collar-type trigon).

Geodesic boundary components of such D are called sides of D.

Theorem 4 (Buser). Surface $\Omega$ can be triangulated into trigons having side lengths $\leq \log 4$ and areas between 0.19 and 1.36 .

Fix such a triangulation; denote by $\mathcal{T}_{c}$ and $\mathcal{T}_{t}$ the sets of its collar-type trigons and ordinary triangles respectively. Also, denote by $\mathcal{S}_{c}$ and $\mathcal{S}_{a}$ the sets of sides of our triangulation which are cycles and geodesic arcs respectively. Let $\mathcal{N}$ be the set of vertices of triangulation. The proof of Theorem 4 from [1] furnishes symmetries of trigons from $\mathcal{T}_{c}$ : namely, $\mathcal{S}_{a}$-sides of such a trigon have equal lengths. From this we derive that lengths of arcs from $\mathcal{S}_{a}$ are bounded from the below by an absolute constant; also, angles of triangulation are also bounded from below by an absolute constant. (For $\mathcal{T}_{t}$-trigons these statements are obvious due to upper area estimate whereas for segments and angles in boundaries of collars the computation is done in [1].)

Lemma 5. If $a_{1}, a_{2}$ are two sides of triangulation with no common vertex, then $\operatorname{dist}_{\Omega}\left(a_{1}, a_{2}\right)$ is bounded from the below by an absolute constant $d_{0}>0$.

Proof. First, notice that distance from any $c \in \mathcal{S}_{c}$ to any other side is bounded from below by a universal constant - otherwise area of some trigon from $\mathcal{T}_{c}$ degenerates.

Next, we claim that distances between vertices of triangulation are bounded from below by a universal constant. Indeed, let $U$ be a metric ball on $\Omega$ centered in some $v \in \mathcal{N}$. If radius of $U$ is small enough then for each $\tau \in \mathcal{T}_{c} \cup \mathcal{T}_{t}$ intersection $U \cap \tau$ can intersect no sides 
of triangulation except for those who emanate from $v$; it is easily checked for both types of trigons, and this leads to our claim.

Now, suppose that $\gamma$ is a geodesic arc joining $a_{1}$ and $a_{2}$ and of small length; it cannot intersect some side from $\mathcal{S}_{c}$ since such sides are far away enough from all the other sides. Suppose that $\gamma$ passes through some trigon $\tau \in \mathcal{T}_{c} \cup \mathcal{T}_{t}$. Then it occurs close enough to some vertex $v \in \mathcal{N}$ (because angles of trigons are bounded from below). Since vertices are separated, the whole curve $\gamma$ is situated close enough to some vertex $v \in \mathcal{N}$, but in this case $\gamma$ can join only sides emanating from $v$. Proof is finished.

Now we estimate Cheeger constants:

Lemma 6. Let $X \subset \Omega$ be a union of $N$ distinct trigons from our triangulation $(N=1,2, \ldots)$. Suppose that $X$ is "connected" in the sense that two trigons are adjacent if they have a common side, not just a vertex. (More formally, we may say that the interior of $X$ is connected.)

Then, under hypothesis on injectivity radius of $\Omega$, we have

$$
h(X) \geq \frac{c(r)}{N} .
$$

Proof. Let $A, B, B^{\prime}$ be sets from definition of Cheeger constant for $X$; we have $A \neq \varnothing$ since $X$ is connected. By Yau lemma ([1], Lemma 8.3.6, see also [5]) we may assume that $B, B^{\prime}$ are connected. If $l(A) \geq r$ then note that $\min \left\{|B|,\left|B^{\prime}\right|\right\} \leq 1.36 \cdot 1 / 2 \cdot N$, and this leads to (1). Next, suppose that $A$ contains a cycle $\gamma$. Then $\gamma$ is homotopic to identity in $\Omega$ (since $l(A)<r$ and by injectivity radius condition). Cycle $\gamma$ should enclose in $\Omega$ a component of area $\leq l(\gamma) / h(\mathbb{H})=l(\gamma)$ (it is known that Cheeger constant of the whole Lobachevskiy plane $\mathbb{H}$ is 1 ) and this also gives (1). So, suppose that $A$ does not contain a cycle.

We could assume from the beginning that $r<d_{0}$ where $d_{0}$ is the constant from Lemma 5 , Set $A$ is a union of curves; take any component $\gamma$ of $A$. Then $\gamma$ necessarily has ends (since $A$ does not contain a cycle) and these ends lie on $\partial X$. Take two of such ends, $p_{1}, p_{2}$, and curve $\gamma_{1} \subset \gamma$ joining them. By Lemma 5, $p_{1}$ and $p_{2}$ are situated either on the same side of triangulation or on two distinct sides emanating from their common vertex; this side or these sides lie on $\partial X$. But if $Y$ is an angle on $\mathbb{H}$ or half-plane of $\mathbb{H}$ then $h(Y)=1$ (see, e.g., proof of Theorem 8.1.2 in [1]). This and also injectivity radius condition, say, in $p_{1}$ lead to (1).

Now, to obtain a subdivision of $\Omega$ via our triangulation, we give a simple graph lemma:

Lemma 7. Let $G$ be a finite connected non-oriented graph with degrees of vertices $\leq 3$. Let $k \in \mathbb{N}$. The set of vertices of $G$ can be subdivided as $V_{1} \sqcup V_{2} \sqcup \cdots \sqcup V_{\alpha} \sqcup V^{\prime}$ (for some $\alpha=0,1,2, \ldots)$ such that:

1. graphs induced by $G$ on each $V_{1}, V_{2}, \ldots, V_{\alpha}, V^{\prime}$ are connected;

2. $2^{k} \leq\left|V_{1}\right|,\left|V_{2}\right|, \ldots,\left|V_{\alpha}\right| \leq 2^{k+1}-1$ and $0 \leq\left|V^{\prime}\right| \leq 2^{k}$.

Proof. We argue by induction by the number of vertices in $G$; for the empty graph the statement is obvious. We may assume that $G$ is a tree. Pick a leaf of $G$ and call it root. Arrange the graph by levels by distance from the root. Vertex $v$ from some level is adjacent to $\leq 2$ vertices from the next level, we call them children of $v$.

Let us construct a sequence of vertices $v_{0}, v_{1}, \ldots, v_{\beta}$ of $G$ ( $\beta$ will be some non-negative integer). Take the root as $v_{0}$. Suppose that $v_{j}$ is constructed and that $v_{l}$ and $v_{r}$ are its children. W.l.o.g., the total number of descendants of $v_{l}$ is greater or equal than that of $v_{r}$. Then put $v_{j+1}:=v_{l}$. If $v_{j}$ has only one child then take it as $v_{j+1}$; and if $v_{j}$ has no children 
then stop our process and put $\beta:=j$, this should occur necessarily. Thus we construct a sequence of vertices.

Now pass this sequence in the reverse order (starting from $v_{\beta}$ and up to $v_{0}$ ) and watch for the total number of descendants of vertices. If $v_{j+1}$ has $x$ descendants (together with itself) then $v_{j}$ has $\leq 2 x+1$ descendants together with itself. Then we have two cases:

1. There exists some $v_{j}$ having $\geq 2^{k}$ and $\leq 2^{k+1}-1$ descendants together with itself. Then, for $V_{1}$ we take the set consisting of $v_{j}$ and of all of its descendants. Cut them from $G$ and apply induction hypothesis for $G$ without $V_{1}$.

2. $|G|<2^{k}$. Then take $V^{\prime}$ as the whole set of vertices of $G$.

Proof of Theorem 1. First, assume that $\varepsilon g \leq 1$. Then we have to prove that $\lambda_{1}>c(r) / g^{2}$, but, by Theorem 2 , it is enough to prove that $h(\Omega)>c(r) / g$. Taking $A$ from the definition of $h(\Omega)$, we see that $A$ must contain a cycle; in this case we argue as in the corresponding case in the proof of Lemma 6 and easily obtain the desired (recall that $|\Omega|=2 \pi(2 g-2)$ ).

Now, suppose that $\varepsilon g>1$. Pick $k \in \mathbb{N}$ with $2^{k} \geq \frac{8 \pi}{0.19 \cdot \varepsilon} \geq 2^{k-1}$, this can be done because $\varepsilon \leq 2$. Let $G$ be the graph of triangulation obtained in Theorem 4: namely, set of vertices of $G$ is $\mathcal{T}_{t} \cup \mathcal{T}_{c}$ and two such trigons are adjacent if they have a common side. Apply Lemma 7 to $G$, take subdivision of the set of vertices of $G$ obtained by this lemma and consider corresponding subdivision of $\Omega$ as $X_{1} \cup X_{2} \cup \cdots \cup X_{\alpha} \cup X^{\prime}$ for some $\alpha=0,1,2, \ldots$ Since trigons have area $\geq 0.19$, we have $\left|X_{j}\right| \geq 2^{k} \cdot 0.19$ for all $j=1,2, \ldots, \alpha$. Then

$$
\alpha \leq \frac{|\Omega|}{2^{k} \cdot 0.19}<\frac{4 \pi g}{2^{k} \cdot 0.19} \leq \frac{\varepsilon g}{2} \leq\lceil\varepsilon g\rceil-1 .
$$

So, $\alpha+1 \leq\lceil\varepsilon g\rceil$. Now, by Lemma [6, we have $h\left(X_{j}\right), h\left(X^{\prime}\right) \geq c(r) / 2^{k}$ for all $j$. This and Theorem 2 lead to the desired.

Finally, let us demonstrate the sharpness of our estimate (we may think that $\varepsilon$ is $1 / k$ ).

Proposition 8. For any $k, l \in \mathbb{N}$ there exists a hyperbolic surface $\Omega$ of genus $k l+1$ with injectivity radius bounded from below by a universal constant and with $\lambda_{l-1}(\Omega) \leq C / k^{2}$, here $C<+\infty$ is a universal constant.

Proof. Let $P$ be fixed hyperbolic pants bounded by geodesic cycles of length, say, 1 (existence and uniqueness of such pants is a well-known fact). Let $P_{1}, \ldots, P_{2 k}$ be copies of these pants. For $j=1,2, \ldots, 2 k$, denote by $\gamma_{1}\left(P_{j}\right), \gamma_{2}\left(P_{j}\right), \gamma_{3}\left(P_{j}\right)$ the boundary components of $P_{j}$. For $j=1,2, \ldots, k$, let us glue $\gamma_{2}\left(P_{2 j-1}\right)$ to $\gamma_{2}\left(P_{2 j}\right)$ and $\gamma_{3}\left(P_{2 j-1}\right)$ to $\gamma_{3}\left(P_{2 j}\right)$. Also, for $j=1,2, \ldots, k-1$ paste $\gamma_{1}\left(P_{2 j}\right)$ to $\gamma_{1}\left(P_{2 j+1}\right)$. Denote by $Q$ the surface obtained in such a way; it is a hyperbolic surface with two geodesic boundary components of length 1 . There exists a Sobolev function $f: Q \rightarrow \mathbb{R}$ with the following properties: first, $f=0$ on $\partial Q$; second, $f$ takes values in $[j-1, j]$ on $P_{j}$ and on $P_{2 k-j+1}$ for $j=1,2, \ldots, k$; third, $|\operatorname{grad} f|$ does not exceed some absolute constant, grad being metric gradient. (To construct such a function, just let it be equal to appropriate constants on boundary components of pants and interpolate it into the interiors of pants anyway.) Now, take $l$ copies of $Q$ and paste them in a cyclic way to obtain a hyperbolic surface $\Omega$ with no boundary. Then genus of $\Omega$ is $k l+1$. Moreover, one can find Sobolev functions $f_{1}, f_{2}, \ldots, f_{l}: \Omega \rightarrow \mathbb{R}$ with disjoint supports and such that $\int_{\Omega} f_{j}^{2} \geq c_{1} \cdot k^{3}, \int_{\Omega}\left|\operatorname{grad} f_{j}\right|^{2} \leq c_{2} \cdot k$ (constants $c_{1}, c_{2}$ are absolute). By the geometric version of minimax principle (that is, by upper estimate from Dirichlet-Neumann bracketing), this leads to the desired eigenvalue estimate. Injectivity radius of $\Omega$ is bounded from the below since it is true for any pants. 


\section{References}

[1] P. Buser, Geometry and Spectra of Compact Riemann Surfaces, Birkhäuser (2010; reprint of 1992 edition).

[2] P. Buser, Cubic graphs and the first eigenvalue of a Riemann surface, Math. Z. 162 (1978), 87-99.

[3] J.-P. Otal, E. Rosas, Pour toute surface hyperbolique de genre $g, \lambda_{2 g-2}>1 / 4$, Duke Math. J. 150, no. 1 (2009), 101-115.

[4] J. Cheeger, A lower bound for the smallest eigenvalue of the Laplacian, in: Problems in analysis, a symposium in honor of S. Bochner, Princeton Univ. Press, Princeton, NJ (1970), 195-199.

[5] S.-T. Yau, Isoperimetric constants and the first eigenvalue of a compact Riemannian manifold, Ann. Sci. Ecole Norm. Sup. 8, no. 4 (1975), 487-507. 